\title{
Time estimation as an index of processing demand in memory search
}

\author{
C. FORTIN \\ Université du Québec, Télé-Université, Québec, Québec, Canada \\ and \\ R. ROUSSEAU \\ Université Laval, Québec, Québec, Canada
}

\begin{abstract}
The influence of cognitive processing on time estimation was investigated. A temporal-interval production and memory-search dual task was devised so that some operations needed by the search took place during the time interval. Subjects were required to produce time intervals concurrently with a memory-search task similar to Sternberg's (1966). On the average, duration increased in proportion to the number of elements in the positive set. In general, temporal-production duration displayed the features previously observed, with speeded responses in memory-search tasks. The additive effect of memory scanning on time estimation made possible an interpretation involving inhibition of timing during concurrent processing in short-term memory. This concurrent processing situation appears to be a fruitful procedure for the study of the interaction between time estimation and cognitive processes. However, since a methodological feature of the search task could favor a successive processing strategy, the possibility that subjects performed the time production and the item-recognition tasks successively makes a conclusive interpretation difficult.
\end{abstract}

Cognitive processes active during a given time interval are known to contribute to the judgment made about its duration (e.g., Michon, 1970). Systematic investigation of the relationship between time estimation and cognitive processes has proven to be a difficult task. A major step in clarifying the issue comes from Hicks, Miller, and Kinsbourne (1976). They reported that under the prospective time-estimation paradigm, in which a subject has to estimate the duration of a completed time period, judged time decreases with increases in amount of other information processing required. This is interpreted to indicate that prospective judgments require attention to time. Concurrent cognitive processing distracts the subject from time processing, thus reducing the amount of temporal information accumulated over an interval; this results in an underestimation of a given physical duration.

In support of this conclusion, Hicks, Miller, Gaes, and Bierman (1977) had subjects perform card sorting or verbal rehearsal for an experimenter-controlled length of time ranging from 8 to $22 \mathrm{sec}$. They showed that verbal estimation of judged time decreased monotonically with the processing demands of the concurrent task, expressed in terms of stimulus uncertainty.

This research was supported by Grant A0692 from the Natural Science and Engineering Research Council of Canada. The authors are grateful to A. B. Kristofferson for his detailed comments on an earlier draft of the paper. Reprint requests should be sent to Robert Rousseau, Ecole de Psychologie, Université Laval, Sainte-Foy, Québec G1K 7P4, Canada.
Brown (1985) also reported an underestimation of the interval in which a subject performed a simple pencilpaper tracking task. However, the effect was not very strong and was limited to conditions in which a 32-sec interval was associated with difficult tracking. No effect was observed with a 16-sec interval. Nevertheless, it can be concluded from these studies that, under the prospective-time-estimation procedure, estimated duration of a given interval becomes shorter with increases in the demands of concurrent cognitive processing.

Other results do not support this claim. In a series of studies, Thomas and his coworkers had subjects judge both the temporal extent of a visual stimulus and some other nontemporal property of the stimulus. Estimated time of the stimulus was shown to increase in proportion to stimulus size (Thomas \& Cantor, 1975), perceived size (Thomas \& Cantor, 1976), and stimulus area (Cantor \& Thomas, 1977). Thomas and Weaver (1975) observed that estimated duration was longer when a visual field contained three letters than when the field was blank. Overestimation was observed when the subject was presented with only the letters; the estimation became larger if he/she was instructed to memorize them.

On the other hand, in Cantor and Thomas (1977), estimated time decreased with increases in stimulus perimeter. Moreover, the effects of both area and perimeter disappeared when stimulus durations were increased from 30 and $70 \mathrm{msec}$ to 500 and $600 \mathrm{msec}$. Finally, Thomas and Cantor (1978) had subjects judge whether a visual display was presented for a "long" 
$(100 \mathrm{msec})$ or a "short" (50 msec) duration and whether it contained the target letter "F." No effect of display size on judged duration was observed when both tasks were performed simultaneously. It is therefore not clear from the work of Thomas and his colleagues whether concurrent processing is likely to have no effect or to produce an increase or decrease in judged duration.

These diverging results are possibly due to the specific conditions under which concurrent processing was studied by Thomas and his coworkers. For instance, the very short durations they used, usually less than $100 \mathrm{msec}$, make it difficult to assume that their subjects were effectively sharing attention between timing and the processing of some other stimulus characteristic. Actually, Long and Beaton $(1980 \mathrm{a}, 1980 \mathrm{~b})$ showed that in Thomas and Weaver's (1975) experimental task, subjects could assess stimulus duration through visual persistence without having to call for a timing process.

Thus, although many studies agree that attention and time estimation are closely related, the precise form of the interaction seems to be determined by the specific experimental conditions assumed to control attentional demands. Since it is doubtful that attentional timesharing can be effectively manipulated under very short durations (under $100 \mathrm{msec}$ ), we might gain a better insight into the relationship between attention and subjective duration by having subjects estimate longer time intervals in the presence of nontemporal processing, somewhat along the line of Hicks and his colleagues (Hicks et al., 1977; Hicks et al., 1976). This approach is further supported by studies in which subjects are requested to fingertap at a rate of one tap per 2 subjective seconds while simultaneously performing some other cognitive processing. In Vroon (1973, Experiment 1) subjects tapped continuously until the occurrence of an auditory stimulus, which was present every $10 \mathrm{sec}$. The subjects were to react as rapidly as possible to the stimulus. Just before stimulus presentation, there was a considerable slowdown in the tapping rate.

In a somewhat similar procedure, Rousseau, Picard, and Pitre (1984) had subjects produce discrete 2-sec intervals. Between 400 and $700 \mathrm{msec}$ after the onset of the interval, a 10-msec tone occurred. The frequency (high or low) of the tone had to be discriminated. When subjects judged that $2 \mathrm{sec}$ had elapsed since the first tap, they terminated the interval by depressing one of two pushbuttons, depending on the frequency of the tone; the discrimination response was postponed until the end of the interval and was combined with the fingertap that marked the end. Produced duration was shown to increase linearly with the length of the delay between the onset of the interval and the tone. It is important to note that overproduction (i.e., lengthening) in Vroon's (1973) and Rousseau et al.'s (1984) tapping situations, corresponds to an underestimation in verbal estimation tasks such as that used by Hicks et al. (1976).

The production method appears particularly interesting because this procedure makes it less likely that judged time is merely a by-product of perceptual processing that is triggered by a stimulus. In both cases, the production performance is linked to changes in the processing of a nontemporal stimulus. The tapping experiments demonstrated that this particular task can be an efficient means for studying the relationship between attention and judged time. Unfortunately, neither of these studies specifically proposed a way to systematically manipulate attention. This leaves some basic questions unanswered. What type of cognitive processing interacts with estimated time? How is time shared between temporal and nontemporal processing?

A psychophysical model ${ }^{1}$ proposed by Rousseau et al. (1984) suggests a systematic approach to the problem. The model proposes that subjects produce an interval by accumulating, after the first tap, a criterion number of pulses emitted by an internal source. The pulses are gated from the emitter through an attentional gate that enables pulse accumulation when it is in an on state. Concurrent cognitive processing that requires attention will put the gate in an off state, thus preventing pulse accumulation. Compared with a situation in which a subject has to produce only time intervals, additional time will be required to reach the criterion number of pulses if time sharing occurs between timing and some other nontemporal processing. This will lead to a lengthening of the produced interval, equal to the total duration of time off.

The present paper describes an attempt to test some of these propositions about the time sharing between concurrent nontemporal and temporal processing. Nontemporal processing demands were manipulated in a memory search (MS) task that was developed by Sternberg (1966). In an MS task, the subject is shown a set of alphanumeric elements, the positive set, and after a short delay is shown a target element, the test item, which is to be recognized as a member or a nonmember of the positive set. Sternberg reported recognition reaction time (RT) to increase linearly with positive-set size. Furthermore, the RT functions had identical slopes, about $35 \mathrm{msec}$, whether the test item was in the positive set (positive trial) or not (negative trial). These results were interpreted as showing MS to be performed through an exhaustive series of mental comparisons between the test item and the positive-set items, comparisons whose durations were independent and additive.

These features of the MS task enable specific predictions to be made about its effect on a concurrent timing process. Assuming that MS and interval production interact according to a kind of preemptive timesharing (Schweickert \& Boggs, 1984) as described by the Rousseau et al. (1984) model, interruption in the timing process by concurrent MS should cause a lengthening in the produced interval that is proportional to the number of mental operations performed in the search and equal to their total duration.

The paradigm designed for the present experiment combined temporal production and MS. The subject was asked 
to produce a 2-sec temporal interval by fingertapping, and the MS was interpolated in the temporal production in the following way. The positive set was memorized before the beginning of the temporal production. Then, $500 \mathrm{msec}$ after the first tap, a test item was presented. The subject terminated the 2-sec interval by depressing one of two pushbuttons, depending on whether the test item did or did not belong to the positive set. The main cognitive operations performed while duration was being estimated were assumed to be the identification of the test stimulus, the comparison of this stimulus with each of the positive-set items, and the decision about the occurrence of a match during the comparison stage.

The basic hypothesis of the present experiment was that memory load, as defined by positive-set size, should have the same effect on temporal productions as it has on reaction time. The duration of the temporal productions should increase linearly with the number of elements in the positive set. The slope of the function should have a value equivalent to that of the RT function, which would reflect the comparison time.

\section{METHOD}

\section{Subjects}

Ten subjects were paid $\$ 5$ per session for their participation. They were young adults who had a mean age of 25 and who were naive to the experimental task.

\section{Apparatus}

The experiment was run in a sound-attenuated test chamber. The subject was seated, with his/her head in a frame that could be fitted at the chin and forehead levels. The subject's right arm rested on a table from which protruded three pushbuttons; when the test stimulus appeared, a positive or a negative response was made by pushing the right or the left button, respectively. A Tektronix Model 602 screen on which the stimuli were presented was about $80 \mathrm{~cm}$ from the subject's eyes. Roughly $10 \mathrm{~cm}$ below the screen, a neon bulb (NE-40) signaled the beginning of a trial in the practice sessions. The positive-set presentation marked the trial's beginning in the experimental sessions. Approximately $15 \mathrm{~cm}$ above the screen was a row of three small lamps which conveyed feedback for the temporal-production task. Accuracy feedback on the detection task was provided through headphones (Realistic PROIIA).

\section{Procedure}

After an introductory session in which the experiment was explained to the subjects, there were four practice and two experimental sessions.

Practice sessions. The first four sessions were designed to stabilize temporal productions and to allow the subject to practice the production and item-detection dual task. These sessions included five blocks. In the first, the subject produced 2-sec intervals. In succeeding blocks, the subject produced the same intervals while simultaneously performing a visual detection task. The trials were run as follows. The subject started the interval by depressing the middle pushbutton. During the interval, a $\mathrm{C}$-shaped stimulus was displayed in the upper or the lower part of the screen; depending on the stimulus location, the subject terminated the temporal production by depressing the right or the left pushbutton, respectively. In the first four blocks of the practice sessions, accuracy feedback on the temporal productions was provided to the subject. The visual signals located above the screen informed the subject that the in-

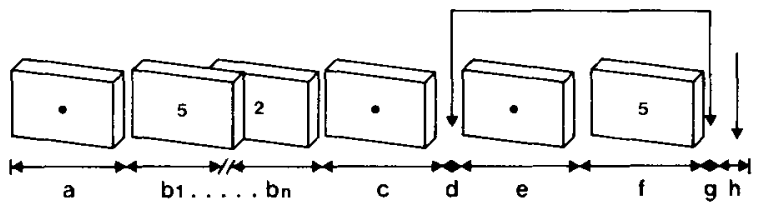

Figure 1. Example of a positive trial: (a) Fixation dot (500 msec); (b) presentation of positive set (1.2 sec per item); (c) fixation dot, which stays on until (d) subject begins the interval; (e) fixation dot (500 msec); (f) test item, displayed until (g) the end of the temporal production; (h) auditive feedback.

terval had been over, under, or within a 200 -msec window centered on the 2-sec standard.

In the last block of the practice sessions, the subjects had to produce the same time interval, terminating the production according to the position of the C-shaped stimulus. In this fifth block, however, no feedback on temporal production was provided.

There were 45 trials per block with a 30 -sec break between blocks.

Experimental sessions. The two experimental sessions were composed of two types of blocks. In the first block of each session, the subject carried out temporal productions combined with a simple visual detection, as had been done in the practice sessions, with feedback on his/her temporal-production performance. The aim of this block was to reset the productions around the target duration. In the next four blocks, the subject performed the experimental task: temporal productions and MS search in a dual-task situation. The MS task, similar to Sternberg's (1966) original version of the MS paradigm, was interpolated in the time-interval production in the following way.

A trial is illustrated in Figure 1. After a central fixation-dot presentation, the positive-set items were successively displayed in the center of the screen. In a positive set, a given item never occurred more than once. The items were presented in a random order for $1.2 \mathrm{sec}$ each without any delay between presentations. The positive set differed from one trial to another in a varied-set procedure. After the last item, the fixation point appeared anew and remained until the subject started the temporal production by depressing the middle pushbutton. The test item was displayed $500 \mathrm{msec}$ after the first buttonpress. The subject's response as to whether the test item did or did not belong to the positive set was withheld until the end of the estimated interval. If the test item was a positive-set member, the subject terminated the interval with a right buttonpress (positive response trial); if the test item was not a positive-set member, the subject terminated the interval with a left buttonpress (negative response trial). After the temporal production, an auditory signal sounded if the trial was positive. The relative frequency of positive and negative trials was equal within a block.

The set of items used in the experiment was composed of 10 different digits $(0,1, \ldots, 9)$. The number of items in the positive set varied from 1 to 6 . As was done in the practice sessions, an auditory signal was presented at the beginning and end of each block. A 30-sec break occurred between blocks. In these experimental sessions, there were 45 trials in the first block and 36 in each of the other four blocks, with 24 trials per positive-set size per subject.

\section{RESULTS}

In the practice sessions, the mean temporal intervals were $1,996 \mathrm{msec}$ in the with-feedback blocks and $2,100 \mathrm{msec}$ in the last without-feedback blocks. In experimental sessions, the mean interval was $2,017 \mathrm{msec}$ for the first block of temporal production alone with feedback. 
An analysis of variance (RBF-6,2,2; Kirk, 1982) was run on the experimental data, that is, for the temporal intervals produced without feedback during the search. Positive-set size, positive-negative trials, and sessions were the main factors. The data from all trials were included in the analysis, since error rates in MS performance were generally quite low, around $1 \%$. Moreover, they were not related to positive-set size or to positive or negative trials.

The results showed the main effect of session successiveness to be significant $[F(1,924)=96.5, p<.0001]$. From the first to the second session, there was a general decrease in mean productions. The productions averaged over all set sizes and response types (positive and negative) show a decrease of $105 \mathrm{msec}$. This main effect is independent of the positive-set size and trial type. The interactions between session successiveness and positiveset size $[F(5,924)=0.73, p=.60]$ and between session and positive-negative trials $[F(1,924)=0.04, p=.85]$ were nonsignificant. Therefore, in spite of betweensessions differences, the absence of an interaction with the other factors makes it possible to average over sessions.

The results further showed a significant main effect of positive-negative response trials $[F(1,924)=18.6$, $p<.0001]$. The negative-trial productions were found to average $46.1 \mathrm{msec}$ longer than positive-trial productions. Finally, a significant main effect of positive-set size $[F(5,924)=11.4, p<.0001]$ was found.

Since significant differences between subjects $[F(9,924)$ $=196.72, p<.0001]$ are explained by differences in mean tapping rate, group data are still meaningful although the positive-set size effect was minimal for 2 subjects.

Figure 2 shows the temporal productions averaged over subjects and over the two experimental sessions plotted against positive-set size. Each point represents $240 \mathrm{ob}-$ servations. It can readily be seen that mean productions increased with positive-set size.

While negative trials displayed a very regular linear trend, the positive-trial function reveals some nonlinear-

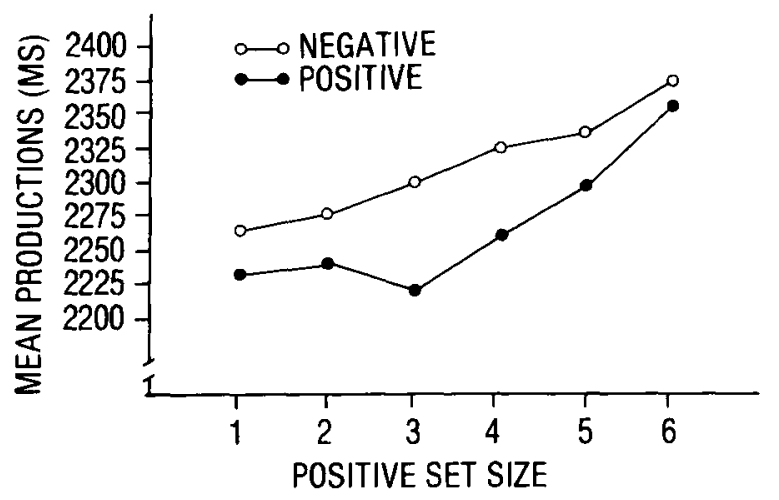

Figure 2. Mean temporal production as a function of positive-set size.
Table 1

Linear Regressions of Mean Productions as a Function of Positive-Set Size on Positive and Negative Trials

\begin{tabular}{lccc}
\hline & Slope & Intercept & $r^{2}$ \\
\hline Positive trials & 23.6 & 2186 & .76 \\
Negative trials & 21.3 & 2240 & .97 \\
\hline
\end{tabular}

ity. Linear regressions, shown in Table 1, account for $97.3 \%$ and $76.0 \%$ of the variance of the mean productions on negative and positive trials, respectively.

The production functions for positive and negative trials have approximately the same slope values: they present a 1:1.1 ratio.

\section{DISCUSSION}

The aim of the present experiment was to test the effect of concurrent cognitive processing on time-interval production. It was proposed that if an MS task was interpolated within a temporal-production task, mean temporalproduction functions should display features comparable to those commonly reported with R'T functions under similar search conditions. Mean production should be a linear function of positive-set size with equal slopes for positive and negative responses.

In a classical item-recognition task, RT functions are known to be linear with a very stable slope, across experiments, of around $35 \mathrm{msec}$ (Sternberg, 1975). This is generally interpreted as an estimate of the time needed to compare the test item with an element of the positive set. In the present task, the slope of the temporalproduction function is about $23 \mathrm{msec}$. There are basically two ways to account for this discrepancy.

First, the discrepancy might be linked to differences among subjects. An examination of the individual data shows that, although the pattern of performance for the majority of our subjects follows closely the trend of the average data, temporal productions for 2 subjects are litthe affected by the concurrent search. Some subjects could have used different timesharing strategies (Pew, 1979). They also may have used completely opposite strategies in the memory-comparison tasks, somewhat in the line of Cooper's (1982) observations with visual search.

The reduced slope may also reflect a much more fundamental characteristic of the timesharing operations. In the Rousseau et al. (1984) model, gating is assumed to be an all-or-none process, such that accumulation of temporal information is completely interrupted by concurrent cognitive processing. Consequently, a one-to-one relationship between cognitive-processing duration and the increase in temporal production was hypothesized. However, if gating were not all-or-none, some temporal information could be accumulated concurrently with search-process execution, and only a certain fraction of the search-process duration would have to be recovered for accumulation purposes. Thus, function relating mean temporal-production duration with positive-set size should display a lower slope. 
The second basic finding is that mean temporal productions were $46 \mathrm{msec}$ longer when the subject reached a negative decision in the search. This result was somewhat unexpected since in the version of the item-recognition paradigm used in the present experiment, RT did not vary with response type (Sternberg, 1966). However, this increase is by no means unique in item-recognition data. With stimuli such as face photographs and nonsense forms, increases of 30 and $55 \mathrm{msec}$, respectively, have been observed in negative-trial RTs (Sternberg, 1969b). Sternberg (1969a) also reported that when the relative frequencies of positive-negative trials were varied, the average negative responses were $45 \mathrm{msec}$ slower than positive responses. Otherwise, in a fixed-set procedure (i.e., when the positive set does not vary from trial to trial), in which positive and negative responses are equiprobable, positive responses were produced $40 \mathrm{msec}$ faster than negative responses, at each set size (Sternberg, 1975).

The present dual task and the fixed-set procedure share one important feature: compared with the RT varied-set procedure, they impose less constraint on the subject. Here, the observed increase in temporal productions could reflect a negative decision duration which, in other more demanding circumstances, might be compressible.

Finally, mean temporal productions did vary significantly over sessions. There was a general decrease in mean production duration from the first to the second session, which could be interpreted as a practice effect. However, the effect of the load as defined by the increase in temporal-production duration in proportion to positiveset size persisted in spite of practice, as indicated by the fact that the variable session showed an insignificant interaction with set size. The reduction in overall load effect of MS on mean time-interval production over sessions is consonant with other findings. Extensive practice of the item-recognition task reduced general mean RT without altering the slope of the function (Kristofferson, 1972).

It should be noted that it is relatively unimportant here that subjects tap at different mean rates, as is the case with the mean RT of subjects in the item-recognition task. The basic interest of the experiment lies in the within-subject or within-group effect on temporal production of load variation in the MS.

The results suggest that the two tasks, temporal production and memory search, interact in a particular way. The somewhat surprising similarity in the overall features of the time-production data and the RT data brings some justification to the a posteriori interpretations that have been proposed. The time-interval-production task may be used as an index of the cognitive operations involved in MS when they are performed concurrently. This dual task appears particularly interesting because it allows the investigation of processes that are usually studied with $R T$ to be examined in new conditions without great time pressure. Moreover, the paradigm developed here does appear to provide a systematic means for studying the interaction between cognitive processing and time estimation. The present data, if interpreted within the context of timesharing, require the identification of the mental resource or function shared by temporal and nontemporal processing. One likely candidate might well be the working memory.

Unfortunately, the exact replication of Sternberg's (1966) search conditions led to a logical difficulty for the assumption that timing and memory search are performed concurrently, since, in Sternberg's study, the test item remained visible until the subject made the buttonpress response. Therefore, there was no need for the subject to process the probe as soon as it was presented.

It would be logically possible, then, to consider the data as the result of a successive execution of the two tasks: the subject completes the timing, and then encodes the test item to perform the search. This could account for the results without the need to infer additive interference caused by the timesharing of a common cognitive process.

A simple evaluation of this possibility could be performed by assuming that the total intertap duration, when positive-set size $=1$, is the sum of the mean production alone without feedback, with the average RT reported by Sternberg (1966) at $n=1$. In the present experiment, the mean temporal production without feedback and without search (i.e., last block of the practice sessions) was 2,100 msec. Moreover, Sternberg showed that the MS of a positive set of one item was about $438 \mathrm{msec}$ for positive trials. Thus, a reasonable estimate of the total timeinterval-production duration resulting from the successive execution of the two tasks is roughly $2,538 \mathrm{msec}$. The actual mean interval productions (positive trial, positive set $=1$ ) is $2,250 \mathrm{msec}$, leaving a difference of 288 -msec. This difference implies either a much shorter RT in the search task or a reduced production adjusted for the successive processing. The $288 \mathrm{msec}$ difference could be reduced by assuming that input and output times are common for the two tasks; however, this still leaves an estimated 100-200-msec difference unaccounted for.

It remains difficult to reach a conclusion on the successive- versus concurrent-processing issue on the basis of the present data. Any conclusive interpretation will have to be delayed until new data are provided under a methodology that reduces the possibility of using a successive-processing strategy. An experiment in which the test item is presented briefly could make an interpretation in terms of a successive-execution strategy much less probable.

\section{REFERENCES}

Brown, S. W. (1985). Time perception and attention: The effects of prospective versus retrospective paradigms and task demands on perceived duration. Perception \& Psychophysics, 38, 115-124.

Cantor, N. E., \& Thomas, E. A. C. (1977). Control of attention in the processing of temporal and spatial information in complex visual pattern. Journal of Experimental Psychology: Human Perception \& Performance, 3, 243-250.

CoOPER, L. H. (1982). Strategies for visual comparison and representation: Individual differences. In R. J. Sternberg (Ed.), Advances in the psychology of human intelligence (Vol. 1), Hillsdale, NJ: Erlbaum. 
Hicks, R. E., Miller, G. W., Gaes, G. \& Bierman, K. (1977). Concurrent processing demands and the experience of time-in-passing. American Joumal of Psychology, 90, 431-446.

Hicks, R. E., Miller, G. W., \& Kinsbourne, M. (1976). Prospective and retrospective judgments of time as a function of amount of information processed. American Journal of Psychology, 89, 719-730.

KIRK, R. E. (1982). Experimental design: Procedures for the behavioral sciences. (2nd ed.). Belmont, CA: Brooks/Cole.

KRISTOFFERSON, M. W. (1972). When item recognition and visual search functions are similar. Perception \& Psychophysics, 12, 379-384.

LONG, G. M., \& BEATON, R. J. (1980a). The contribution of visual persistence to the perceived duration of brief targets. Perception \& Psychophysics, 28, 422-430.

LONG, G. M., \& BEATON, R. J. (1980b). The effects of spatial frequency and target type on perceived duration. Perception \& Psychophysics, 28, 413-421.

Michon, J. A. (1970). Processing of temporal information and cognitive theory of time experience. Studium Generale, 23, 249-265.

PEw, R. W. (1979). Secondary tasks and workload measurement. In N. Moray (Ed.), Mental workload, its theory and measurement (pp. 23-28). New York: Plenum Press.

Rousseau, R., Picard, D., \& Pitre, E. (1984). An adaptive counter model for time estimation. Annals of the New York Academy of Sciences, 423, 639-642.

SCHWEICKERT, R., BogGS, G. J. (1984). Models of central capacity and concurrency. Journal of Mathematical Psychology, 28, 223-281.
STeRnBerG, S. (1966). High-speed scanning in human memory. Science, $153,652-654$

STERNBERG, S. (1969a). The discovery of processing stages: Extensions of Donders' method. Acta Psychologica, 30, 276-315.

STERNBERG, S. (1969b). Memory scanning: Mental processes revealed by reaction-time experiment. American Scientist, 57, 421-457.

STERNBERG, S. (1975). Memory scanning: New findings and current controversies. Quarterly Joumal of Experimental Psychology, 27, 1-32.

Thomas, E. A. C., CANTor, N. E. (1975). On the duality of simultaneous time and size perception. Peception \& Psychophysics, 18 44-48.

Thomas, E. A. C., CANToR, N. E. (1976). Simultaneous time and size perception. Perception \& Psychophysics, 19, 353-360.

Thomas, E. A. C., \& CANTor, N. E. (1978). Interdependence between the processing of temporal and non-temporal information. In J. Requin (Ed.), Attention and performance VII (pp. 43-62). Hillsdale, NJ: Erlbaum.

Thomas, E. A. C., \& Weaver, W. B. (1975). Cognitive processing and time perception. Perception \& Psychophysics, 17, 363-367.

VROON, P. A. (1973). Tapping rate as a measure of expectancy in terms of response and attention limitation. Joumal of Experimental Psychology, 101, 183-185.

(Manuscript received March 17, 1986; revision accepted for publication April 23, 1987.)

\section{Notice}

\section{SPECIAL ISSUE \\ PSYCHOBIOLOGY \\ CALL FOR MANUSCRIPTS}

A special issue of Psychobiology is being planned in the area of Psychobiology of Sexual Differentiation and Gender-Related Behaviors. It will include both empirical research and review papers. Manuscripts should review empirical literatures, advance theory or present major sets of data. Publication of the special issue is planned for Fall 1988. The deadline for initial submissions is March 1, 1988.

Contributions intended for the special issue should be sent to the guest editor: Jane Stewart, Center for Studies in Behavioral Neurobiology, Concordia University, 1455 de Maisonneuve Blvd. West, Montreal, PQ H3G 1M8, Canada. 